\title{
Influence of soft ferromagnetic sections on the magnetic flux density profile of a large grain, bulk Y-Ba-Cu-O superconductor
}

\author{
M P Philippe ${ }^{1}$, M D Ainslie ${ }^{2}$, L Wéra ${ }^{1}$, J-F Fagnard ${ }^{1}$, A R Dennis ${ }^{2}$, Y-H Shi ${ }^{2}$, D A Cardwell ${ }^{2}$, \\ B Vanderheyden ${ }^{1}$ and $\mathrm{P}$ Vanderbemden ${ }^{1}$ \\ ${ }^{1}$ SUPRATECS and Department of Electrical Engineering and Computer Science (B28), \\ University of Liège, 4000 Liège, Belgium \\ ${ }^{2}$ Bulk Superconductivity Group, Engineering Department, University of Cambridge, \\ Cambridge CB2 1PZ, UK \\ E-mail: M.Philippe@ulg.ac.be
}

\begin{abstract}
Bulk, high temperature superconductors have significant potential for use as powerful permanent magnets in a variety of practical applications due to their ability to trap record magnetic fields. In this paper, soft ferromagnetic sections are combined with a bulk, large grain $\mathrm{Y}-\mathrm{Ba}-\mathrm{Cu}-\mathrm{O}$ (YBCO) high temperature superconductor to form superconductor/ferromagnet (SC/FM) hybrid structures. We study how the ferromagnetic sections influence the shape of the profile of the trapped magnetic induction at the surface of each structure and report the surface magnetic flux density measured by Hall probe mapping. These configurations have been modelled using a 2D axisymmetric finite element method based on the $\boldsymbol{H}$-formulation and the results show excellent qualitative and quantitative agreement with the experimental measurements. The model has also been used to study the magnetic flux distribution and predict the behaviour for other constitutive laws and geometries. The results show that the ferromagnetic material acts as a magnetic shield, but the flux density and its gradient are enhanced on the face opposite to the ferromagnet. The thickness and saturation magnetization of the ferromagnetic material are important and a characteristic ferromagnet thickness $d^{*}$ is derived: below $d^{*}$, saturation of the ferromagnet occurs, and above $d^{*}$, a weak thicknessdependence is observed. The influence of the ferromagnet is observed even if its saturation magnetization is lower than the trapped flux density of the superconductor. Conversely, thin ferromagnetic discs can be driven to full saturation even though the outer magnetic field is much smaller than their saturation magnetization.
\end{abstract}

Keywords: bulk (RE)BCO superconductors, magnetic measurements, numerical modelling, trapped field magnets, ferromagnet

PACS: 74.25.Ha Magnetic properties; 74.72.-h Cuprate superconductors; 75.60.Ej Magnetization curves, hysteresis, Barkhausen and related effects; 74.25.Qt Vortex lattices, flux pinning, flux creep 


\section{Introduction}

Bulk, high temperature superconductors have significant potential for use as powerful permanent magnets in a variety of practical applications due to their ability to trap record magnetic fields. The typical trapped field in bulk superconductors $\left(>3 \mathrm{~T}\right.$ in bulk $\mathrm{MgB}_{2}$ [1-4] or $>17 \mathrm{~T}$ in bulk $(\mathrm{RE}) \mathrm{Ba}_{2} \mathrm{Cu}_{3} \mathrm{O}_{7}$ large grain materials $[5,6]$ ) is well beyond the saturation magnetization of conventional ferromagnets. This makes them extremely promising as a competing technology for traditional permanent magnets in various applications [7-10]. The combination of ferromagnetic and superconducting materials can enhance the performance of the superconductor $[11,12]$ and even lead to new applications [13]. Large grain, bulk superconductors are often used in applications that incorporate ferromagnetic materials, such as in motors and generators [14, 15]. Ferromagnets can also increase the force in levitation systems $[16,17]$ and close the magnetic circuit, which improves the available flux produced by bulk superconductors [18]. Similarly, ferromagnetic materials are used as sheaths around multifilament wires and tapes $[19,20]$ or as magnetic flux diverters to modify the flux distribution around tapes and superconducting coil magnets [21-28]; thereby improving their electrical properties (i.e. increasing the critical current and reducing AC losses).

Ferromagnets can be used for enhancing the trapped flux in applications in which bulk superconductors are used as permanent magnets. In our previous work [29], we characterized the increase of the remanent volume average flux density due to the presence of ferromagnet sections attached to one side of the superconductor for various configurations. We also determined how the maximum flux density (i.e. at the centre of one of the faces) is modified. In the present work, we investigate how ferromagnetic sections combined with a bulk, large grain Y-Ba-Cu-O (YBCO) superconductor influence the profile shape of the trapped magnetic induction available at the surface. Without the ferromagnet, the magnetic flux distribution above a bulk superconducting magnet is strongly non-uniform (conical profile predicted by the Bean model $[30,31])$. The purpose of the present study is to determine how ferromagnets can be used to shape this magnetic induction and improve the flux uniformity. Knowing that the saturation magnetization of ferromagnets is physically limited to around $2 \mathrm{~T}$ or less, another question is to determine whether the ferromagnets can still be used in a regime where the trapped flux density in the superconductor exceeds this value. In order to answer to these questions, we first use magnetic flux density Hall probe mappings and numerical modelling to investigate the interaction between a fully magnetized bulk superconductor (SC) and ferromagnets (FM) of different sizes and shapes, placed on one face of the bulk material. The magnetic properties of both the ferromagnetic material (hysteresis cycle) and the superconductor (critical current density $J_{\mathrm{c}}(B), n$-value) were determined from independent experiments and are known accurately. The finite element method, based on the 2D axisymmetric $\boldsymbol{H}$-formulation is used to recreate the observed measurement results and understand in detail how the ferromagnetic components modify the magnetic flux density above each side of the SC/FM assembly. The excellent agreement with the measurements validates the model, which is then used in the second part of the paper to predict the magnetic behaviour in other cases. We study the influence of $(i)$ the thickness of the ferromagnet, (ii) its saturation properties, and (iii) the superconductor properties (critical current density $J_{c}$ ) on the magnetic flux distribution.

\section{Materials}

\subsection{Superconducting sample}

A solid, cylindrical bulk YBCO superconductor of diameter $16.5 \mathrm{~mm}$ and height $6.32 \mathrm{~mm}$, with its $c$-axis parallel to its thickness, was synthesized using conventional top seeded melt growth (TSMG) at the University of Cambridge (UK). The melt-processed, large grain microstructure consists of a 
superconducting $\mathrm{YBa}_{2} \mathrm{Cu}_{3} \mathrm{O}_{7-\delta}(\mathrm{Y}-123)$ phase matrix containing discrete $\mathrm{Y}_{2} \mathrm{BaCuO}_{5}(\mathrm{Y}-211)$ inclusions $[32,33]$. The top and bottom faces of the as-processed grain were polished prior to characterization.

The magnetic characterization of this sample at $77 \mathrm{~K}$ was presented in our previous work [29, 34]. The field dependent critical current $J_{\mathrm{c}}(B)$ is experimentally found to follow a Bean-Kim law $J_{\mathrm{c}}(B)=$ $J_{\mathrm{c} 1}\left(1+|\boldsymbol{B}| / B_{1}\right)^{-1}$ with $J_{\mathrm{c} 1}=13.8 \mathrm{kA} / \mathrm{cm}^{2}$ and $B_{1}=0.987 \mathrm{~T}$. The time-dependence of the remnant magnetization follows a power law $B \propto\left(1+t / t_{0}\right)^{1 /(1-n)}$ with $n=45$ for $t \gg t_{0}$.

\subsection{Ferromagnetic materials}

The ferromagnetic sections used in the measurements were machined from the soft ferromagnetic alloy "Supra50" [35], which contains mostly iron and nickel (51.5 wt $\%$ and $47 \mathrm{wt} \%$, respectively). Please note that the name Supra50 is not related to superconductivity. Its intrinsic magnetic hysteresis $B(H)$ curve was presented in [29]. In this work, the alloy is characterized by two main parameters: the maximum relative permeability $\mu_{\mathrm{r}, \max }$ and the saturation magnetization $M_{\mathrm{sat}}$ whose values are summarized in table 1 . The coercive field of the Supra50 alloy was found experimentally to be 520 $\mathrm{A} / \mathrm{m}$ and will be neglected in this work. The cross-sections of the investigated structures are shown in figure 1 together with the dimensions of the machined ferromagnetic sections. The D1 and D2 configurations are made with a $0.99 \mathrm{~mm}$ and $1.90 \mathrm{~mm}$ thick ferromagnetic disc, respectively. An "inverted cone" is used in the IC configuration; its thickness is $2.88 \mathrm{~mm}$ at the edge and decreases linearly to a residual thickness of $0.09 \mathrm{~mm}$ in $r=0$. The RG configuration is made with a $2.88 \mathrm{~mm}$ thick ferromagnetic ring which has a $9.52 \mathrm{~mm}$ internal diameter. The ferromagnet has the same external diameter as the superconductor $(16.5 \mathrm{~mm})$ in all the measured configurations.

Two other commercial soft ferromagnetic materials were used in the numerical modelling: "Permimphy" from Aperam [35] and "Vacoflux" from Vacuumschmelze [36]. Their magnetic properties, summarized in table 1, were obtained from our measured data for Permimphy and through the manufacturer specifications for Vacoflux. Again, the coercive field of these materials is neglected.

Table 1. Characteristics of the ferromagnetic materials: saturation magnetization $\mu_{0} M_{\text {sat }}$ and maximum differential permeability $\mu_{\mathrm{r}, \max }$.

\begin{tabular}{lcc}
\hline Material & $\mu_{0} M_{\text {sat }}$ & $\mu_{\mathrm{r}, \max }$ \\
\hline Supra50 & $1.4 \mathrm{~T}$ & $1.7 \times 10^{3}$ \\
Permimphy & $0.8 \mathrm{~T}$ & $2.0 \times 10^{3}$ \\
Vacoflux & $2.2 \mathrm{~T}$ & $1.7 \times 10^{3}$ \\
\hline
\end{tabular}

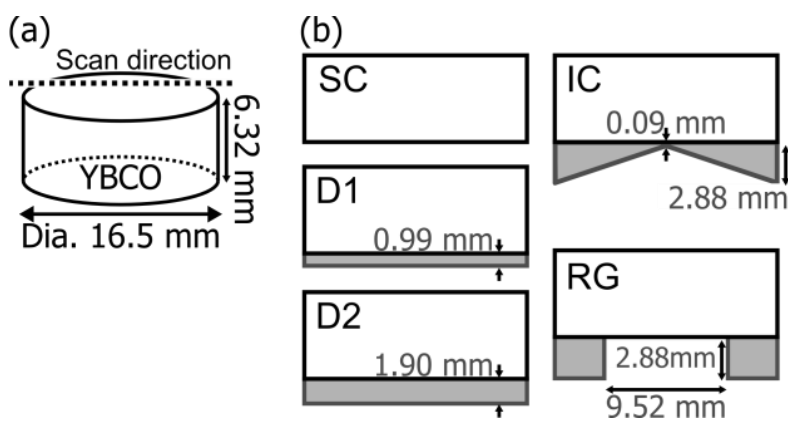

Figure 1 (a) Dimensions of the YBCO sample and scan direction along the diameter of the sample. (b) Crosssections and dimensions of the measured SC/FM configurations. The ferromagnet (grey) is made of Supra50 and has the same external diameter as the superconductor $(16.5 \mathrm{~mm})$ in all the measured configurations. 


\section{Experimental setup}

The distribution of magnetic flux density above the surface of the SC/FM hybrid structure was investigated by Hall probe mapping [37, 38]. The vertical component of magnetic flux density above the magnetized sample is mapped with a miniature Hall probe (AREPOC AXIS-3H) controlled by a $\mathrm{XYZ}$ micropositioning stage. The probe is placed $0.5 \mathrm{~mm}$ above the measured surface of the whole $\mathrm{SC} / \mathrm{FM}$ hybrid structure. The bottom coating under the probe is estimated to be $0.35 \mathrm{~mm}$ thick, which means that the flux density is measured approximately $0.85 \mathrm{~mm}$ above the highest point of the measured surface. A full mapping corresponds to a $30 \mathrm{~mm}$ wide square with $1 \mathrm{~mm}$ long steps. The sample and the SC/FM hybrid structures are magnetized along the superconductor's $c$-axis using the field cooling (FC) method under a uniform field of $670 \mathrm{mT}$ produced by an iron-cored electromagnet, which ensures full magnetization of the sample. The mapping procedure always starts 20 minutes after the magnetizing field has been removed to minimize the influence of flux relaxation. The sample is fully immersed in liquid nitrogen $(77 \mathrm{~K})$ during the whole experiment (magnetization and subsequent measurement). For the hybrid SC/FM configurations, the ferromagnet was present during the field cooled magnetization (and not added after the magnetization process).

\section{Modelling framework}

Numerical modelling of superconducting materials is a powerful tool to help explain experimental results, investigate the physical mechanisms underlying the observed behaviour and to predict the performance of the SC/FM structures under analysis in this work. The numerical model developed here combines the electromagnetic equations governing the behaviour of the superconductor based on the 2D axisymmetric $\boldsymbol{H}$-formulation [39-42] with the same modifications made to include magnetic subdomains with a relative permeability $\mu_{\mathrm{r}}(H)$ as described in [25, 26, 43, 44].

The model is implemented in the commercial FEM software package COMSOL Multiphysics 4.3a [45] using the general form partial differential equation (PDE) interface, and the governing equations are derived from Maxwell's equations - namely, Faraday's (1) and Ampere's (2) laws:

$$
\begin{gathered}
\nabla \times \boldsymbol{E}+\frac{\mathrm{d} \boldsymbol{B}}{\mathrm{d} t}=\nabla \times \boldsymbol{E}+\frac{\mathrm{d}\left(\mu_{0} \mu_{r} \boldsymbol{H}\right)}{\mathrm{d} t}=0 \\
\nabla \times \boldsymbol{H}=\boldsymbol{J}
\end{gathered}
$$

where $\boldsymbol{H}=\left[H_{\mathrm{r}}, H_{\mathrm{z}}\right]$ represents the magnetic field components, $\boldsymbol{J}=\left[J_{\phi}\right]$ represents the current density and $\boldsymbol{E}=\left[E_{\phi}\right]$ represents the electric field. $\mu_{0}$ is the permeability of free space, and for the superconducting and air subdomains, the relative permeability is simply $\mu_{\mathrm{r}}=1$. For the ferromagnet subdomains, an appropriate constant value for $\mu_{\mathrm{r}}$ is used, resulting in a linear $B(H)$ curve, until the saturation value $\mu_{0} M_{\text {sat }}$ is reached, based on the values given in table 1 . Thus, $\mu_{\mathrm{r}}$ is represented in the numerical simulation by the following equation:

$$
\mu_{\mathrm{r}}= \begin{cases}\mu_{\mathrm{r}, \mathrm{max}} & \text { for } B<\mu_{0} M_{\text {sat }} \\ \left(1+\frac{M_{\mathrm{sat}}}{H}\right) & \text { for } B \geq \mu_{0} M_{\text {sat }}\end{cases}
$$

A Kim-model [46] approximation for the critical current density $J_{\mathrm{c}}$ is assumed for the in-field behaviour of the bulk, as described in section 2.1, and the electrical properties are modelled using a non-linear $E-J$ power law [47, 48], $E \propto J^{n}$, where $n=45$. 
Since the magnetization process is slow, isothermal conditions are assumed; hence, no thermal model is included. In order to simulate the field cooled procedure used in the experiment (as experimentally performed in [29]), a zero field cooled process is employed in the simulation, and based on a simple Bean model $[30,31]$ approximation, as long as the externally applied field is twice the full penetration field of the bulk sample, the trapped field will be the same as in the case of field cooling [49]. A ramped, external magnetizing field up to $3 \mathrm{~T}$ is applied to the SC/FM structure, then removed, over a period $T=400 \mathrm{~s}$ (i.e. $B_{\text {ext }}=3 \mathrm{~T}$ at $t=200 \mathrm{~s}$ ), by applying appropriate boundary conditions in the model [39].

\section{$5 \quad$ Results and discussion}

\subsection{Measurement results}

In this section, we investigate the influence of the ferromagnet on the profile of the magnetic field distribution on both sides of the SC/FM assemblies for different geometric configurations through Hall probe mappings and modelling.

Figure 2(a) shows the measured magnetic induction $B_{\mathrm{z}} 0.85 \mathrm{~mm}$ above each of the faces of the superconductor alone (SC configuration). The induction follows a conic profile on both faces as predicted by the Bean model [30, 31]. The field on the top face (containing the seed) is found to be approximately $34 \%$ higher than on the bottom face (far from the seed). This fact is due to the unavoidable radial and axial distribution of $T_{\mathrm{c}}$ and $J_{\mathrm{c}}$ in bulk, large grain superconductors and results typically in a higher $J_{\mathrm{c}}$ in the layer of the superconductor located near the seed [29, 38, 50-52]. A reduced $T_{\mathrm{c}}$ is mainly observed near the seed and results from contamination from the seed crystal. This reduced $T_{\mathrm{c}}$ might, however, be compensated by an increased irreversibility field in this region. The critical current density variations results from variations of the local microstructure and of the influence of the seed contamination [50]. In addition, the critical current density is usually found to be higher at the boundaries between the growth sectors than in these growth sectors [10]. Note that some inhomogeneities of the material studied in this work were probed experimentally in our previous work [29].

Figure 2(b) shows the mappings $0.85 \mathrm{~mm}$ above each face for the D2 hybrid configuration, in which a $2 \mathrm{~mm}$ thick ferromagnetic Supra50 disc is placed under the superconductor. On the top face, the presence of the ferromagnet leads to a $6.5 \%$ increase of the maximum flux density, as presented in table 2. On the bottom face, an important change in the shape of the flux density occurs: the flux density distribution exhibits now a plate-like shape and its value on the symmetry axis is strongly decreased.

The results shown in figure 2 are consistent with those obtained in our previous work [29] where the measured quantities were (i) the average volume flux density and (ii) the flux density probed by a fixed Hall probe stuck at the centre of both circular faces. The results can be explained as follows. The attenuation of the maximum flux density on the bottom face results from a shielding effect of the ferromagnet, which acts as a magnetic short-circuit and drives the flux lines directly towards the edges of the superconductor. To a first approximation, the increase of maximum flux density on the top face can be understood through the image theorem, since flux lines must be perpendicular to the ferromagnet/superconductor interface on the superconductor side. The image theorem predicts that adding a semi-infinite volume (extending to infinite radius and depth) of perfectly ferromagnetic material $\left(\mu_{\mathrm{r}} \rightarrow \infty\right)$ is equivalent to doubling the height of the superconducting cylinder. A superconducting cylinder with a higher aspect ratio experiences a lower demagnetizing field and has a 
larger magnetic flux density at its surface. In practice, the real effect is not as significant as the image theorem would predict, since the ferromagnet is not semi-infinite. Since the results plotted in figure 2 exhibit clear axisymmetric behaviour, we can examine in detail the ferromagnet-induced modifications of the flux density distribution by plotting measurements radially along any diameter of the assembly, which is shown in figure 3(a).

(a) Superconductor alone

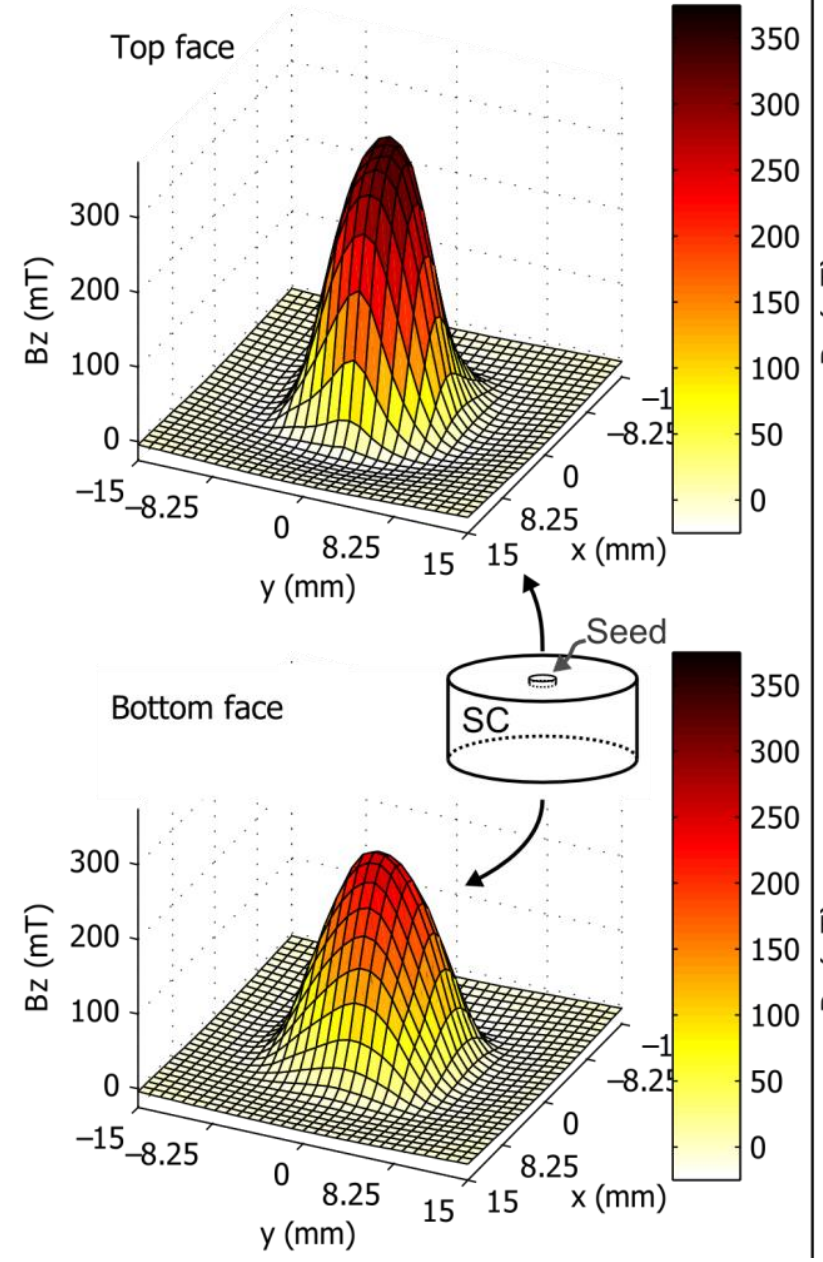

(b) D2 configuration

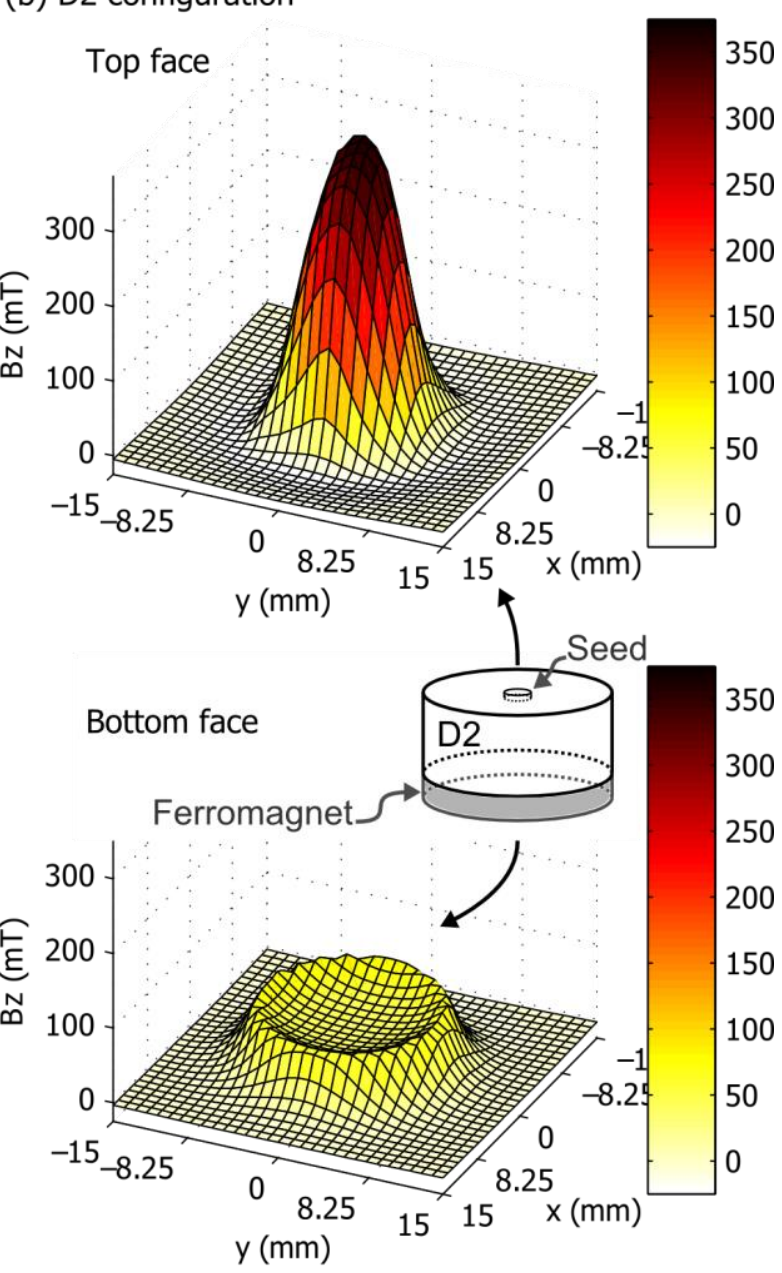

Figure 2 Hall probe mappings of $B_{z}$, the vertical component of the magnetic flux density generated by the fully magnetized bulk high temperature superconductor. (a) The superconductor only; (b) A $1.90 \mathrm{~mm}$ thick ferromagnetic disc is placed below the HTS and acts as a shield. In each case, mappings are performed $0.85 \mathrm{~mm}$ above the external surface of the whole hybrid structure.

Figure 3(a) shows the radial measurements of $B_{\mathrm{z}}$ for the measured configurations presented in figure 1 . It is immediately apparent that the maximum flux density occurs at a slightly off-centre position. The position corresponding to the measured maximum on the bottom face in the absence of ferromagnet $(r$ $=-1 \mathrm{~mm})$ was selected as the reference position. The characteristic values $B_{\mathrm{z}}^{*}=B_{\mathrm{z}}(r=-1 \mathrm{~mm})$ are summarized in table 2 , together with their relative variation compared to the SC alone. On the top face (i.e. opposite to the ferromagnet), figure 3(a) shows an increase of $B_{z}$ for each hybrid SC/FM configuration. This increase ranges between $2.1 \%$ and $8.0 \%$ depending on the shape of the ferromagnet. On the bottom face (i.e. close to the ferromagnet), the distribution of $B_{z}$ changes from a 
conical to a plate-like shape when the ferromagnet covers the entire surface (D1, D2, and IC). The flux density is the largest for a ring aligned with the edges of the ferromagnet. The ferromagnetic ring (RG) does not cover the entire surface of the superconductor and the measured flux density exhibits three maxima with a central "bump". It is of interest to note that a larger increase in $B_{z}$ on the top face corresponds to a larger decrease on the bottom face in all measured configurations.

These flux distribution measurements are consistent with the results obtained in previous work [29]. A thicker ferromagnetic disc leads to a larger increase in magnetic flux on the face opposite to the ferromagnet. For a given volume of ferromagnet, the shape also plays an important role since the inverted cone (IC) enables a larger flux modification than the disc (D2). The flux distribution on the ferromagnet side is strongly shape-dependent: when the ferromagnetic material does not cover the centre of the surface, the magnetic flux density arising from the supercurrent located under the uncovered zone is not shielded, leading to a succession of minima and maxima. Although such a peculiar shape is not relevant to the present study, it shows the possibility to modulate the induction using a succession of holes and ferromagnetic sections. Such a "nearly sinusoidal" flux distribution is desired in some applications, e.g. strong field undulators or wigglers to be used in future synchrotron light sources and e-e linear colliders [53].

\section{(a) Experiment}

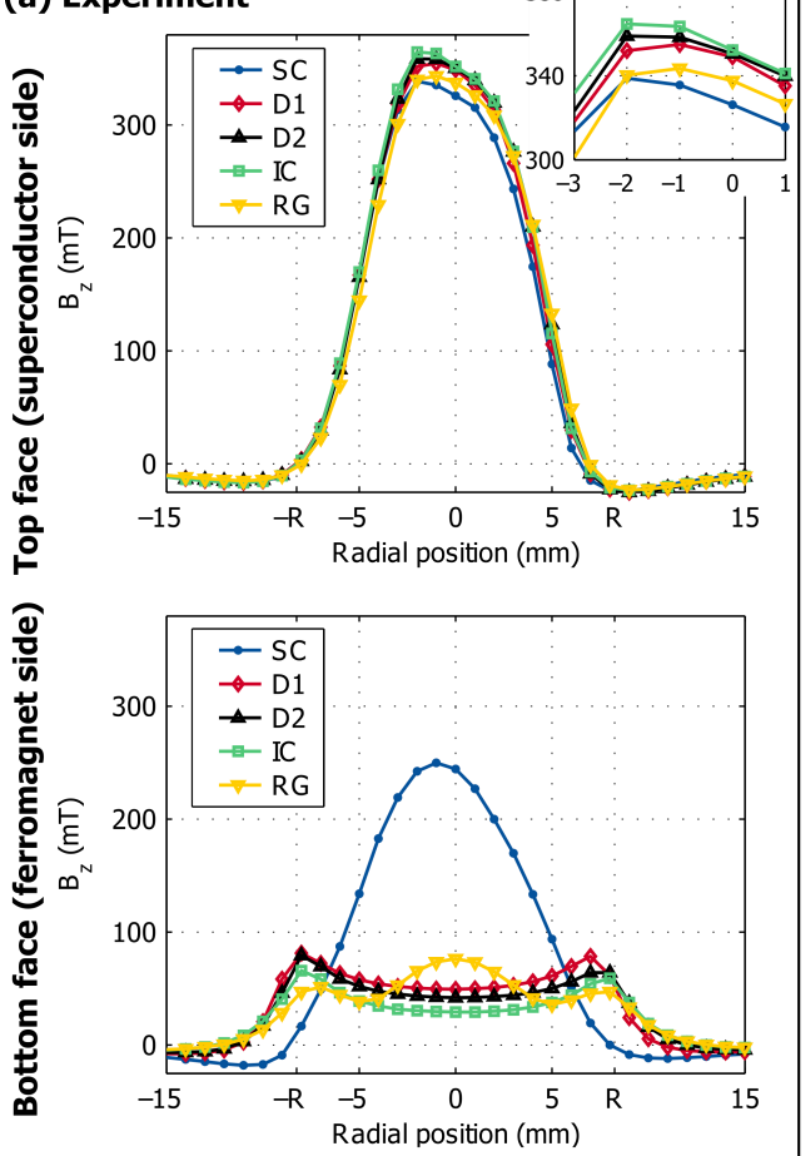

(b) Modelling
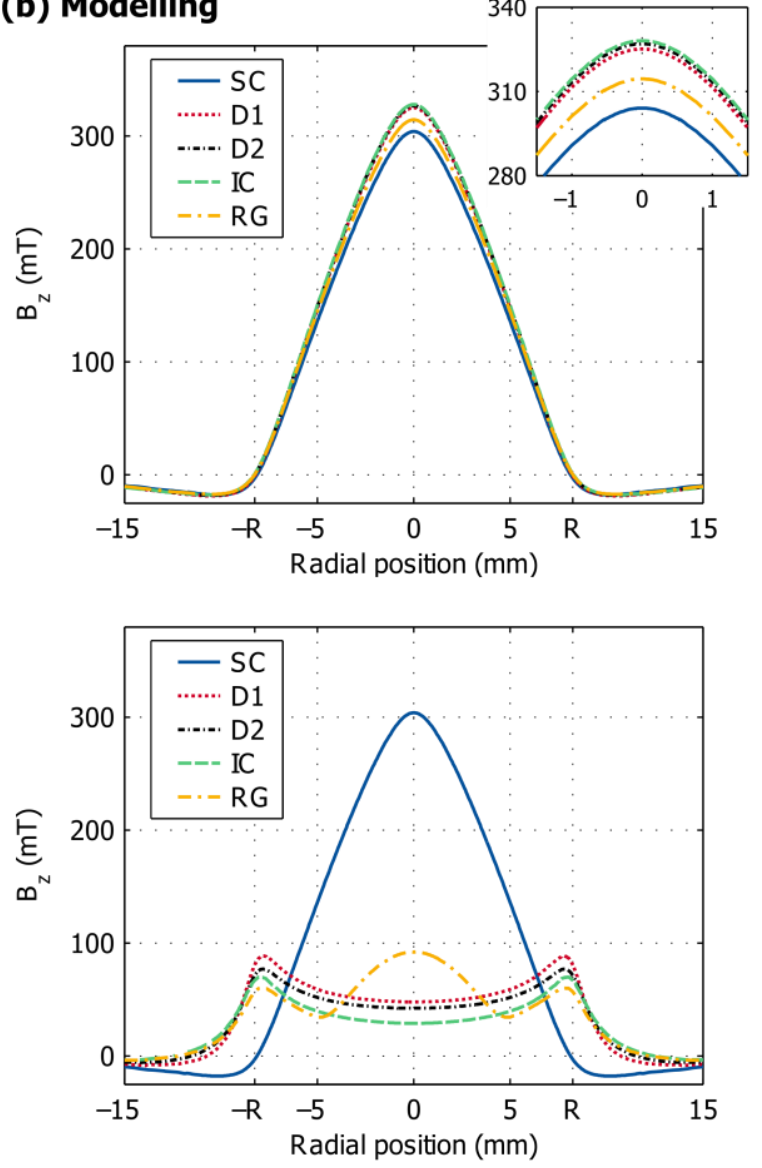

Figure 3 (a) Magnetic flux density $B_{\mathrm{z}}$ above the top and bottom surfaces of the SC/FM hybrid structures shown in figure 1. All measurements are performed along a diameter $0.85 \mathrm{~mm}$ above the external surface of the whole hybrid structure. $R=8.25 \mathrm{~mm}$ is the radius of the SC/FM assembly. (b) Modelling results for the same SC/FM structures. 
Table 2. Numerical values of the remanent magnetic flux density $B_{\mathrm{z}}$ shown in figure 3 . (a) $B_{\mathrm{z}}^{*}=B_{\mathrm{z}}(r=$ $-1 \mathrm{~mm}$ ) for the measurement results and (b) $B_{\mathrm{z}}^{\mathrm{c}}$ at the centre for modelling. All values are taken $0.85 \mathrm{~mm}$ above the external surface of the whole hybrid structure. The variations relative to the superconductor alone (SC) configuration are presented for both measurements and modelling.

\begin{tabular}{|c|c|c|c|c|}
\hline \multirow[b]{2}{*}{$\begin{array}{l}\text { Top face } \\
\text { Configuration }\end{array}$} & \multirow{2}{*}{$\begin{array}{c}\text { (a) Measurement } \\
B_{\mathrm{z}}^{*}(\mathrm{mT})\end{array}$} & \multicolumn{3}{|c|}{ (b) Modelling } \\
\hline & & $\begin{array}{l}\text { Relative } \\
\text { variation }\end{array}$ & $B_{\mathrm{z}}^{\mathrm{c}}(\mathrm{mT})$ & $\begin{array}{l}\text { Relative } \\
\text { variation }\end{array}$ \\
\hline $\mathrm{SC}$ & 336 & -- & 304 & -- \\
\hline D1 & 355 & $5.7 \%$ & 325 & $6.9 \%$ \\
\hline D2 & 358 & $6.5 \%$ & 327 & $7.6 \%$ \\
\hline IC & 363 & $8.0 \%$ & 328 & $7.9 \%$ \\
\hline RG & 343 & $2.1 \%$ & 314 & $3.3 \%$ \\
\hline \multicolumn{5}{|l|}{ Bottom face } \\
\hline Configuration & $B_{\mathrm{z}}^{*}(\mathrm{mT})$ & $\begin{array}{l}\text { Relative } \\
\text { variation }\end{array}$ & $B_{\mathrm{z}}^{\mathrm{c}}(\mathrm{mT})$ & $\begin{array}{l}\text { Relative } \\
\text { variation }\end{array}$ \\
\hline $\mathrm{SC}$ & 250 & -- & 304 & -- \\
\hline D1 & 49.7 & $-80.1 \%$ & 48.0 & $-84.2 \%$ \\
\hline D2 & 42.5 & $-83.0 \%$ & 42.4 & $-86.1 \%$ \\
\hline IC & 29.7 & $-88.1 \%$ & 29.0 & $-90.5 \%$ \\
\hline $\mathrm{RG}$ & 73.6 & $-70.6 \%$ & 92.0 & $-69.7 \%$ \\
\hline
\end{tabular}

\subsection{Modelling of the measured configurations}

In this section, the modelling framework presented in section 4 is used, together with the parameters introduced in section 2, to model the magnetic flux distribution of all configurations investigated experimentally. Figure 3(b) shows the modelling results corresponding to the measurements shown in Figure 3(a), i.e. the distribution of $B_{\mathrm{z}}$ along the diameter, $0.85 \mathrm{~mm}$ above each surface. Table 2(b) gives the numerical values at $r=0$ and their variations relative to the superconductor alone (SC) configuration.

It can be seen that the modelled curves shown in figure 3(b) exhibit mirror symmetry at $r=0$ (centre of the bulk) since they are obtained from a 2D axisymmetric model. In the absence of any ferromagnet (SC configuration), the results are identical on the top and bottom faces since the model assumes uniform, macroscopic $J_{\mathrm{c}}(B)$ properties for the bulk superconductor. This is in contrast with experimental results where some $J_{\mathrm{c}}(r, z)$ dependence needs to be considered to account for the different flux distributions on top and bottom faces [10]. Since the $J_{\mathrm{c}}(B)$ data used in the modelling were obtained from non-destructive average magnetic measurements over the whole bulk pellet [29, 34], the modelled distribution results are an intermediate between those measured on the top and bottom faces, as expected intuitively.

As can be seen in figure 3, there is an excellent qualitative agreement between the measurements and modelling results. Remarkably, the exact characteristics of the experimental flux profiles can be reproduced accurately with the model. On the top face, i.e. opposite to the ferromagnetic sections, the sequence of all modelled plots is the same as for the experiment. Quantitatively, the relative variations of $B_{\mathrm{z}}$ at the reference position, as summarized in table 2, are similar. These relative variations differ for a maximum of 1.5 percentage points (pp) on the top face and $4.1 \mathrm{pp}$ on the bottom face.

The nice agreement between the experimental and numerical results provides strong evidence that our model is able to reproduce the magnetic properties of a superconducting disc with different ferromagnetic sections, the properties of both being obtained by preliminary independent experiments. 
This gives confidence that this model can be used to study the flux redistribution and predict the influence of magnetic materials of other properties and/or shapes on the trapped field produced by large, bulk high temperature superconductors.

\subsection{Modelling results for other configurations}

Following validation of the model by experiment, this can now be used to study new configurations where the parameters can be changed one at a time. Additionally, quantities not directly available through measurements (e.g. the distribution of the flux density $B$ inside the materials) are extracted from modelling results. In this section, we will first study geometric and magnetic modifications of the ferromagnet, including their influence on the gradient of $B_{\mathrm{z}}$. Next, the influence of the critical current density of the superconductor will be examined. In particular, we will investigate the regime for which the maximum flux density produced by the superconductor exceeds the saturation magnetization of the ferromagnet.

5.3.1 Influence of the disc thickness. Figure 4 shows the modelled induction obtained on each face for SC/FM structures using different ferromagnetic discs made of Supra50 material. In addition to the D1 and D2 configurations introduced in section 2, three new configurations are investigated: D035, D070, and D3, corresponding to disc thicknesses of $0.35 \mathrm{~mm}, 0.70 \mathrm{~mm}$, and $2.90 \mathrm{~mm}$, respectively. All discs have the same diameter as the superconductor. To make the comparisons easier, the crosssections of these configurations are shown on the right-hand side part of figure 4. The SC-alone configuration is also shown for reference.

On the top face, a thicker disc on the bottom gives a higher flux density compared to the reference sample. The $0.70 \mathrm{~mm}$ and thicker discs lead to a similar increase while the $0.35 \mathrm{~mm}$ thick disc is experimentally found to be much less efficient. A similar redistribution for the different discs can be observed on the bottom face: the plate-like shape of the flux density is obtained for the $0.70 \mathrm{~mm}$ and thicker discs, while the $0.35 \mathrm{~mm}$ thick disc exhibits a completely different behaviour. In the latter, the flux distribution is intermediate between that obtained for the superconductor alone and for the thicker discs and displays a centred peculiar "camel-bump". Actually, a closer look at the D070 curve shows the emergence of this bump as well (see inset of figure 4). For thicker discs, the flux density at a constant distance $(0.85 \mathrm{~mm})$ from the ferromagnet is a decreasing function of the ferromagnet thickness. The relative increase of $B_{\mathrm{z}}^{\mathrm{c}}=B_{\mathrm{z}}(r=0)$ on the top face as a function of the thickness $d$ of the ferromagnet is shown in figure 5. The curve displays a "kink" occurring at some disc thickness $d$ " within the range of about $0.7 \mathrm{~mm}$ to $1.0 \mathrm{~mm}$. The increase is strongly thickness-dependent for $d<d^{*}$ and then flattens for $d>d^{*}$. A similar "kink" was also observed in the same range of ferromagnet thicknesses at scan heights of $0.1 \mathrm{~mm}$ and $1.5 \mathrm{~mm}$, which indicates that the "kink" is independent of the scan height. The origin of this behaviour is discussed below. 

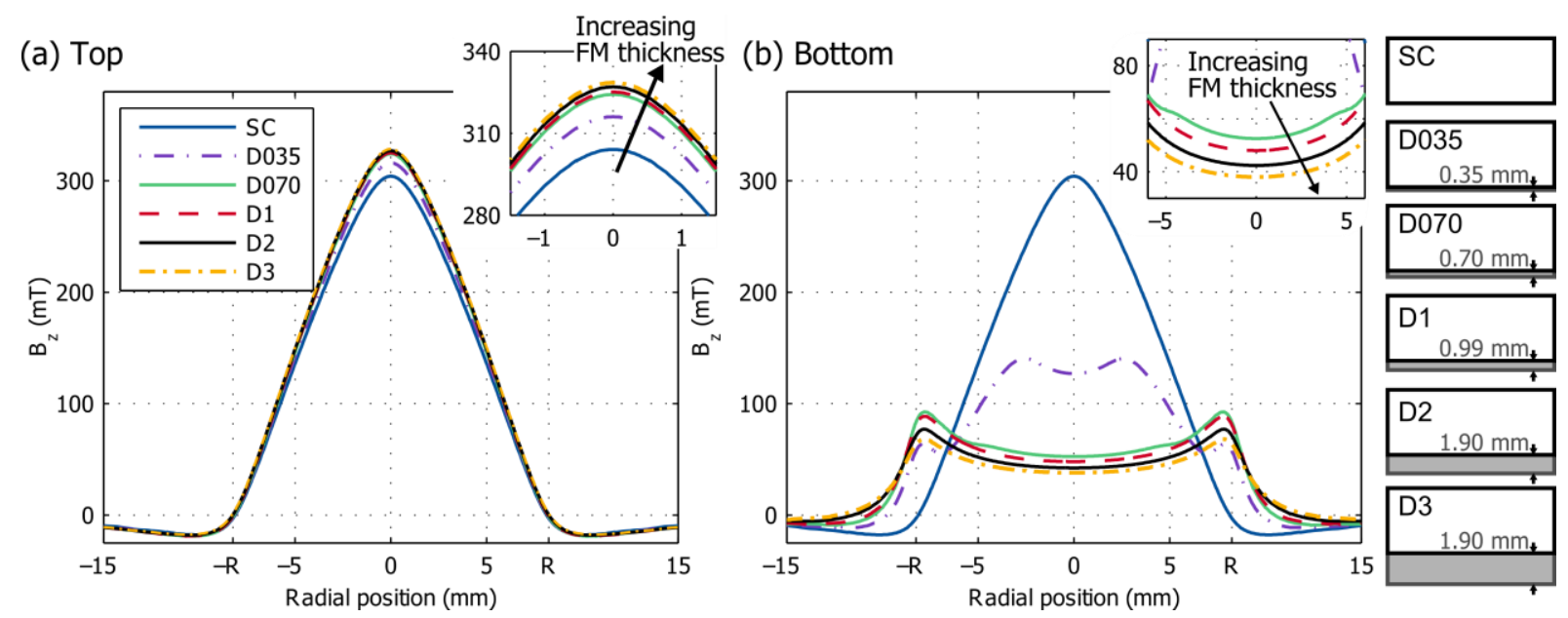

Figure 4 Influence of FM disc thickness on $B_{z}$, modelling $0.85 \mathrm{~mm}$ (a) above and (b) under the SC/FM structures sketched on the right. On the sketches, the FM discs and their thicknesses are in grey. The thickness of the superconductor is $6.32 \mathrm{~mm}$.

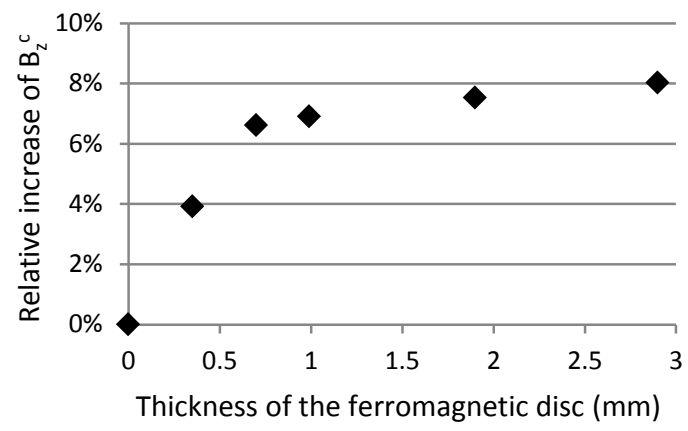

Figure 5 Relative increase of $B_{\mathrm{z}}^{\mathrm{c}}=B_{\mathrm{z}}(r=0)$ on the top face of the superconductor, as a function of the thickness of the ferromagnetic disc attached to the opposite face.

In order to obtain additional information about the particular D035 and D1 configurations (i.e. on both sides of the kink), it is of interest to examine the distribution of magnetic flux lines generated by the magnetized superconductor. These are shown in figure 6 . The $0.99 \mathrm{~mm}$ disc (D1) is found to drive a very large proportion of the flux lines towards the edges of the superconductor. This disc is not fully saturated, as evidenced from the colour scale which does not reach the saturation magnetization of 1.4 T. This behaviour is also observed on the D2 and D3 configurations (figures not shown). The 0.35 $\mathrm{mm}$ disc (D035), however, is nearly fully saturated. The saturation magnetization is reached in a majority of the ferromagnet volume; only small regions near the symmetry axis and near the outer face of the ferromagnet are not saturated. 

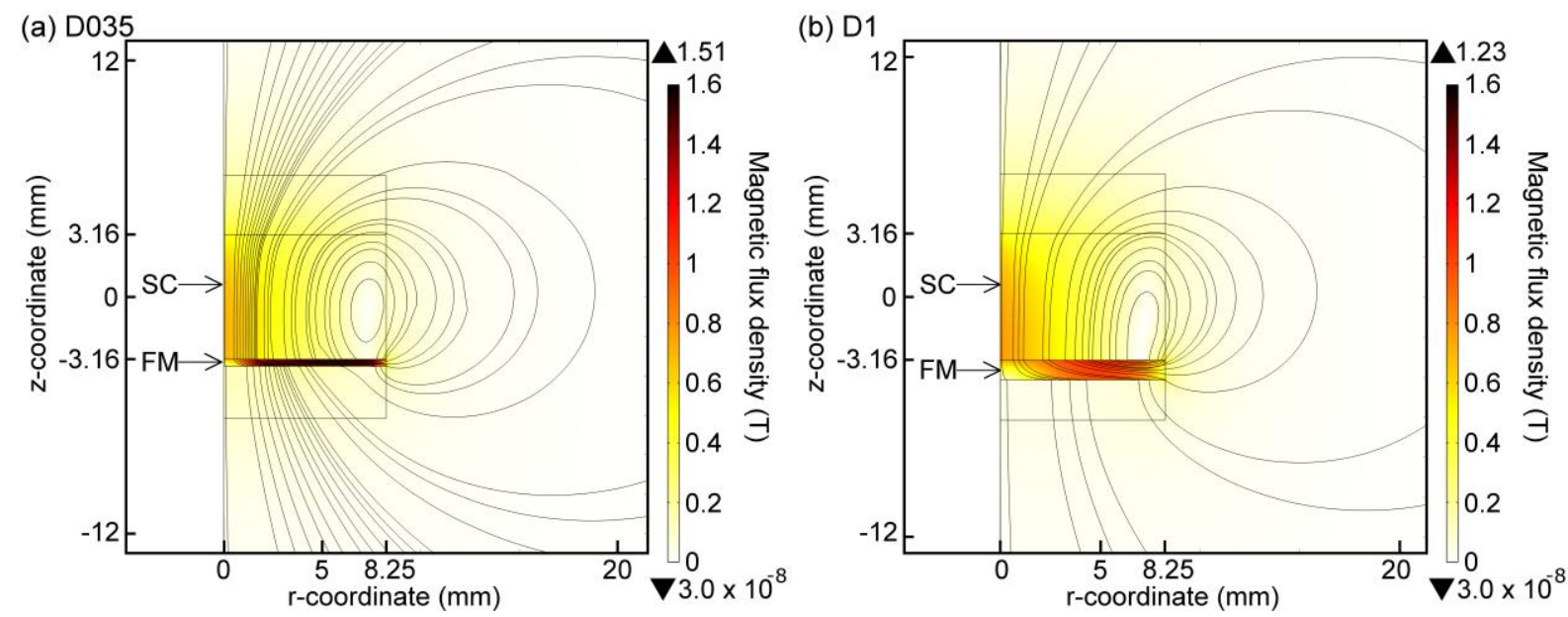

Figure 6 Magnetic flux density plots of the modulus of $B$ (colour scale) for the (a) D035 and (b) D1 configurations. The flux lines are a guide to the eye to show the direction of the local flux density. The ferromagnetic discs are made from Supra50 material, characterized by a saturation magnetization $\mu_{0} M_{\text {sat }}=1.4 \mathrm{~T}$.

The remarkable feature of the above results is that a large volume of the ferromagnetic disc is brought close to saturation $(B \approx 1.4 \mathrm{~T})$, although the maximum flux density that would exist against the superconductor without ferromagnet is $\approx 0.3 \mathrm{~T}$, i.e. one fourth of the saturation magnetization of the ferromagnet. The saturation is responsible for the much different behaviour of the $0.35 \mathrm{~mm}$ disc, compared to thicker ones. The kink occurring in figure 5 is thus related to the "threshold" thickness $d$ " under which this saturation occurs. It is obviously linked to $(i)$ the superconductor generated flux and therefore to its size and critical current - and to (ii) the saturation magnetization of the ferromagnetic material. In order to extend the generality of these results to other sizes and ranges of physical properties, it is tempting to roughly estimate this "threshold" thickness $d^{*}$ from a simplified analysis based of conservation of magnetic flux and to compare it to the interval of about 0.7 to 1.0 $\mathrm{mm}$ found in figure 5. First, we assume that the superconducting disc (radius $a$ ) is characterized by a field-independent $J_{\mathrm{c}}$, i.e. the true $J_{\mathrm{c}}(B)$ is replaced by a constant $J_{\mathrm{c}}$ producing almost the same flux distribution above the superconductor alone. Note that, as will be shown below (section 5.3.3), the corresponding field-independent $J_{\mathrm{c}}$ in the present case is $J_{\mathrm{c} 2}=1.03 \times 10^{8} \mathrm{~A} / \mathrm{m}^{2}$. As a first approximation, the radial dependence of flux density in the median plane $(\mathrm{z}=0)$ is given by $B(r)=$ $\mu_{0} J_{\mathrm{c} 0}(a-r)$. If we further assume that there are no stray field lines through the lateral surface of the superconductor, the total flux $\phi$ generated by the superconductor is given by radial integration of $B(r)$, i.e. $\phi=\mu_{0} J_{\mathrm{c} 0} \pi a^{3} / 3$. In the case of a perfect (infinitely permeable) ferromagnet, axial magnetic flux lines exiting the superconductor at the SC/FM interface would be channelled by the ferromagnet along the radial direction, i.e. they would exit the ferromagnet through its lateral surface. For a ferromagnetic disc of thickness $d$, conservation of magnetic flux imposes therefore that the magnetic flux density in the ferromagnet $B_{\mathrm{FM}}$ is such that

$$
B_{\mathrm{FM}}(2 \pi a d)=\mu_{0} J_{c 0}\left(\pi a^{3} / 3\right)
$$

A rough approximation of the flux density in the ferromagnet is therefore given by

$$
B_{\mathrm{FM}}=\mu_{0} J_{\mathrm{c} 0}\left(a^{2} / 6 d\right) .
$$

Since this hypothetical, perfect (infinitely permeable) behaviour occurs only when the magnet is not saturated, the minimum ferromagnet thickness required (denoted $d^{*}$ ) is such that 


$$
\begin{gathered}
B_{\mathrm{FM}}=\mu_{0} J_{\mathrm{c} 0}\left(a^{2} / 6 d\right)<\mu_{0} M_{\mathrm{sat}} \\
d>\left(J_{\mathrm{c} 0} a^{2} / 6 M_{\mathrm{sat}}\right)=d^{*}
\end{gathered}
$$

Using the actual parameters $\left(J_{\mathrm{c} 0} \approx 10^{8} \mathrm{~A} / \mathrm{m}^{2}, \mu_{0} M_{\mathrm{sat}}=1.4 \mathrm{~T}, a=8.2510^{-3} \mathrm{~m}\right)$, we obtain a threshold thickness given by $d^{*} \approx 1 \mathrm{~mm}$. This value, estimated with rather "crude" assumptions, is in fair agreement with the interval found using finite element modelling $(0.7$ to $1.0 \mathrm{~mm})$. As a consequence, formula (3) can be used as a simple design rule to determine the minimum required thickness of ferromagnetic material. Note that for a given superconductor geometry and a given critical current, increasing the ferromagnet thickness above $d^{*}$ will not impact significantly on the surrounding field.

More precisely, we can derive an analytical upper bound for the relative increase of $B_{\mathrm{z}}^{\mathrm{c}}$ on the superconductor side when a ferromagnet of the same radius is attached to the opposite face. This upper bound is the expected horizontal asymptote of the graph shown in figure 5 when the ferromagnet is a perfect ferromagnetic material occupying the semi-infinite volume $z<0$. The expected increase for an infinitely thick ferromagnetic "disc" is likely to be bounded by the increase that would occur for a semi-infinite ferromagnet. Since the latter configuration is equivalent to doubling the height of the superconducting disc, we can roughly estimate the effect of the ferromagnet from the analytical formula giving the magnetic flux density along the axis of a fully magnetized superconducting cylinder (radius $a$, height $L$, critical current density $J_{\mathrm{c}}$ ) $[10,54,55]$

$$
B_{\mathrm{z}}(z)=\frac{1}{2} \mu_{0} J_{\mathrm{c}}\left\{\left(z+\frac{L}{2}\right) \ln \left(\frac{a+\sqrt{a^{2}+(z+L / 2)^{2}}}{|z+L / 2|}\right)-\left(z-\frac{L}{2}\right) \ln \left(\frac{a+\sqrt{a^{2}+(z-L / 2)^{2}}}{|z-L / 2|}\right)\right\}
$$

where $z$ is the elevation from the centre of the superconductor. At the centre of one face of the superconductor $(z=L / 2)$, the flux density $B_{\mathrm{CF}}$ is equal to

$$
B_{\mathrm{CF}}=B_{\mathrm{z}}\left(\frac{L}{2}\right)=\frac{1}{2} \mu_{0} J_{\mathrm{c}} L \ln \left[\frac{a+\sqrt{a^{2}+L^{2}}}{L}\right]
$$

which can be rewritten as a function of the dimensionless (thickness / radius) ratio $\zeta=L / a$, i.e.

$$
B_{\mathrm{CF}}=\frac{1}{2} \mu_{0} J_{\mathrm{c}} a f(\zeta)
$$

where the function $f(\zeta)$ is introduced, given by

$$
f(\zeta)=\zeta \ln \left[\frac{1}{\zeta}+\sqrt{1+\frac{1}{\zeta^{2}}}\right]
$$

The theoretical increase that would be due to a semi-infinite ferromagnet is therefore given by $[f(2 \zeta)-f(\zeta)] / f(\zeta)$. For the geometric parameters of the superconductor investigated in this work $(L$ $=6.32 \mathrm{~mm}, a=8.25 \mathrm{~mm}, \zeta=0.766$ ), one obtains $\approx 13 \%$. This rough upper bound is indeed a few percent above the relative increase modelled for the thickest ferromagnet investigated (8\%). The conclusion to be drawn from this analysis is that a ferromagnet is extremely helpful in increasing the flux density of a superconductor, but that a thickness much larger than the threshold value $d^{*}$ defined above is not needed. 
Since many applications of bulk superconductors are linked to levitation systems [56-58], it is also of interest to investigate whether a ferromagnetic disc placed at the bottom of the superconducting pellet is beneficial to the vertical levitation force above the superconductor top face. Along the axis of the superconductor, this levitation force is proportional to the gradient of flux density $\mathrm{d} B_{z} / \mathrm{d} z[59,60]$. Figure 7 shows the modelled magnetic induction $B_{\mathrm{z}}$ along the symmetry axis (i.e. for $r=0$ ) for the D035 and D1 configurations. The $z$ derivative of these curves can be used to study the influence of the ferromagnet on the levitation force. The modelling results show that the addition of the ferromagnetic disc on one side increases $\mathrm{d} B_{z} / \mathrm{d} z$ at $r=0$ on the opposite side by $3.1 \%$ and $5.2 \%$ with the $0.35 \mathrm{~mm}$ and $0.99 \mathrm{~mm}$ discs, respectively (values taken $0.85 \mathrm{~mm}$ above the superconductor). Although the relative increase of $\mathrm{d} B_{\mathrm{z}} / \mathrm{d} z$ is not as high as the relative increase in $B_{\mathrm{z}}$, it can be concluded that the presence of the ferromagnet yields an increase of the levitation force above the superconducting pellet.

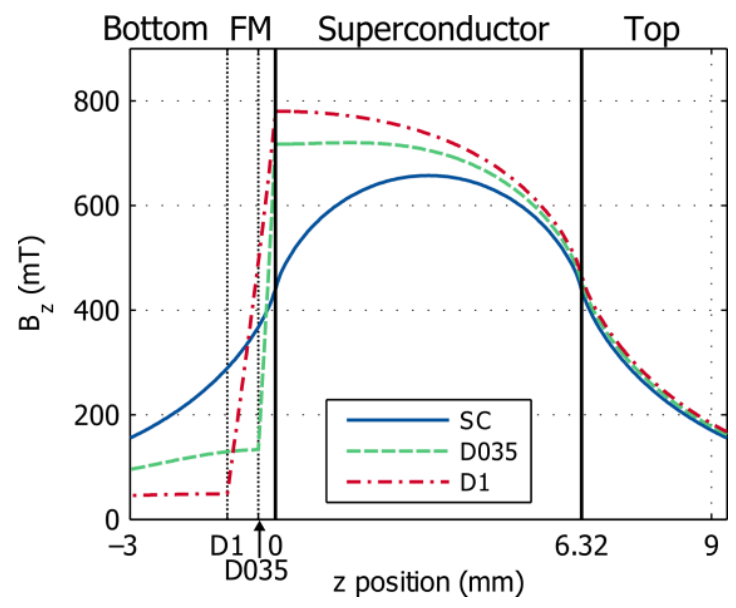

Figure 7 Magnetic induction $B_{\mathrm{z}}$ along the symmetry axis $(r=0)$ of the D035 and D1 configurations. The superconductor alone (SC) is shown for reference. The vertical continuous lines show the limits of the superconductor. The D035 and D1 abscissae show the limit of the ferromagnet in the D035 and D1 configurations, respectively.

5.3.2 Influence of the type of ferromagnetic material. Figure 8 shows the flux distribution modelled above the D035 configuration for three ferromagnetic materials of different saturation magnetizations: Supra50 $\left(\mu_{0} M_{\text {sat }}=1.4 \mathrm{~T}\right.$; violet dash-dot $)$ as shown above, Permimphy $\left(\mu_{0} M_{\text {sat }}=0.8 \mathrm{~T}\right.$; red dash $)$, and Vacoflux $\left(\mu_{0} M_{\text {sat }}=2.2 \mathrm{~T}\right.$; green line). The magnetic induction obtained for the superconductor alone is shown for reference (blue line). On the bottom face, the three $B_{z}$ profiles exhibit several bumps. When increasing the saturation magnetization $M_{\text {sat }}$ of the ferromagnetic material, the general trend is that $B_{z}$ decreases in the central region and increases near the edges of the sample. At the highest saturation magnetization investigated, the shape tends to the plate-like shape observed for thicker ferromagnetic discs. On the top face, the maximum flux density is an increasing function of saturation magnetization $M_{\text {sat }}$ of the ferromagnet. The corresponding flux density increase, as a function of $M_{\text {sat }}$, is shown in figure 9. A quasi-linear behaviour is observed in the studied range. These results are in agreement with those obtained when varying the thickness of the ferromagnet (section 5.3.1): when the ferromagnet is saturated, only a small fraction of the flux lines can be channelled radially towards the lateral surface of the ferromagnet. According to equation (3), the threshold thickness below which saturation occurs and the "bumps" in $B_{\mathrm{z}}$ appear - is inversely proportional to the saturation magnetization $M_{\text {sat }}$. This means that for $\mu_{0} M_{\text {sat }}=2.2 \mathrm{~T}$, this threshold thickness would be approximately $64 \%$ of that for the Supra50 studied earlier. Qualitatively, increasing the saturation magnetization of the soft ferromagnet has the same effect globally as increasing its thickness. The conclusion to be drawn is that 
ferromagnets with the highest saturation magnetization possible are always preferred, but that the intrinsic (physical) limitation of the saturation magnetization of classical ferromagnets can be overcome by using thicker ferromagnetic discs.
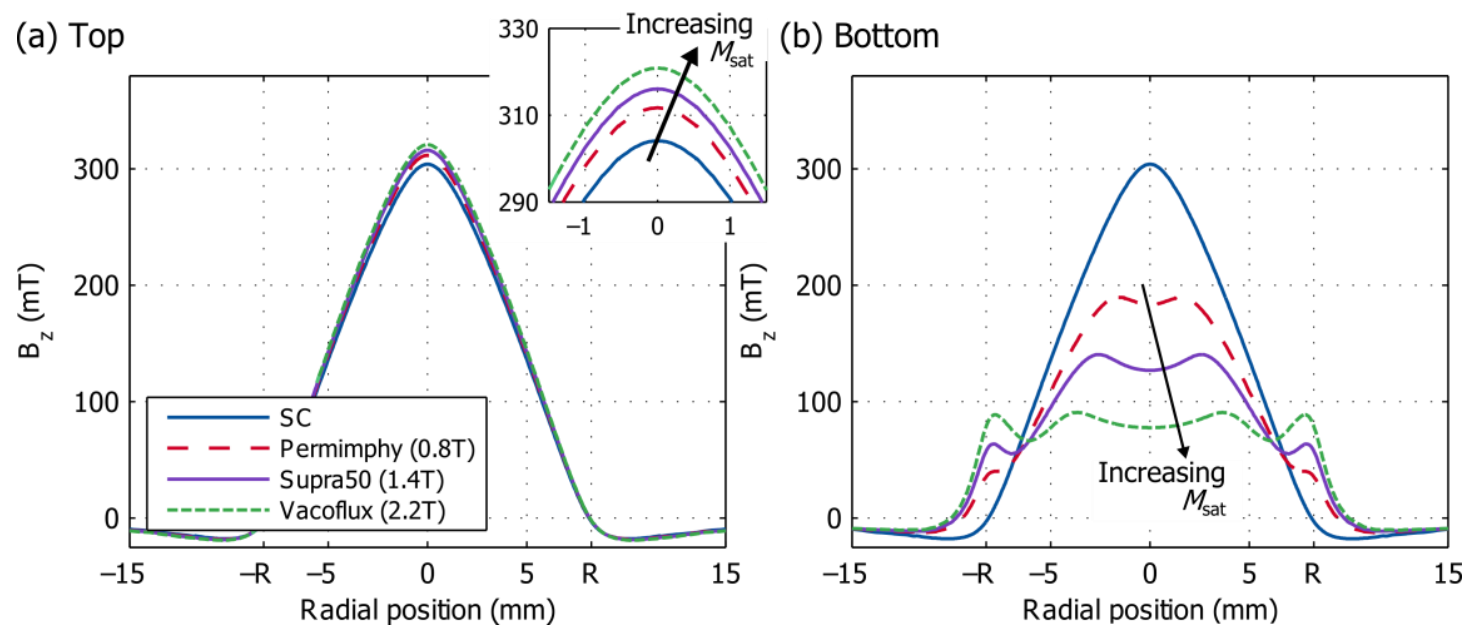

Figure 8 Magnetic induction $B_{\mathrm{z}}$ above the top and bottom surfaces for the D035 configuration $(0.35 \mathrm{~mm}$ thick disc) for three different saturation magnetizations. Three materials are compared: $\operatorname{Supra} 50\left(\mu_{0} M_{\mathrm{sat}}=1.4 \mathrm{~T}\right.$; violet dash-dot) as presented before, Permimphy $\left(\mu_{0} M_{\text {sat }}=0.8 \mathrm{~T}\right.$; red dash), and Vacoflux $\left(\mu_{0} M_{\text {sat }}=2.2 \mathrm{~T}\right.$; green line). The superconductor alone is shown for reference (blue line).

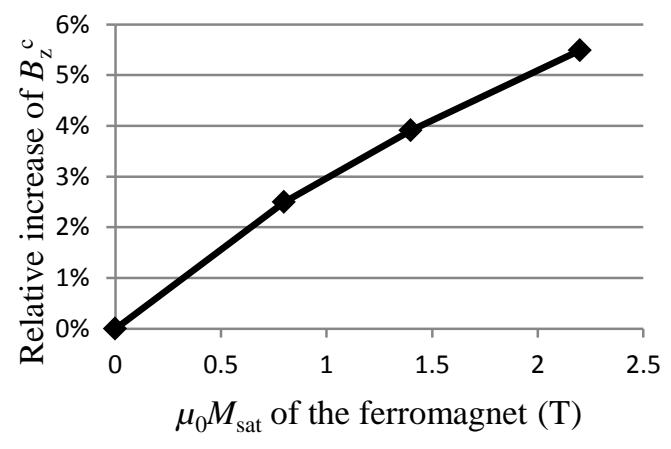

Figure 9 Relative increase of $B_{\mathrm{Z}}^{\mathrm{c}}$ at the centre of the top face of the superconductor as a function of the saturation magnetization of the ferromagnetic material placed on the bottom face.

5.3.3 Importance of the critical current. The numerical model is used in this section to study the influence of the critical current density of the superconductor on the above results. We first compare the magnetic flux profiles obtained either with a field-dependent $J_{\mathrm{c}}(B)$ or with a constant $J_{\mathrm{c}}$. Next, we investigate the properties of a large- $J_{c}$ hybrid structure generating a field much higher than the saturation magnetization of the ferromagnet.

Figure 10 shows the flux distribution above the different hybrid SC/FM structures with a constant critical current $J_{\mathrm{c} 2}=1.03 \times 10^{8} \mathrm{~A} / \mathrm{m}^{2}$. This particular "average" constant $J_{\mathrm{c}}$ is used since the $B_{\mathrm{Z}}$ profile is nearly the same as that obtained with original $J_{\mathrm{c}}(B)$ law for the SC configuration (superconductor alone). Remarkably, the constant and field dependant critical currents lead to similar distributions of $B_{z}$ on both the bottom and top faces of the SC/FM assemblies. The practical conclusion is the following: although the superconducting material is characterized by some field- 
dependent $J_{\mathrm{c}}(B)$, this $J_{\mathrm{c}}(B)$ dependence is not so crucial for investigating the flux profiles at some distance for the hybrid SC/FM structures. Note that the regime investigated here is one for which the flux density in the superconductor is on average $50-60 \%$ of the $B_{1}$ parameter of the $J_{\mathrm{c}}(B)$ law, which means that field-induced variations of the critical current density can indeed be found in the superconducting volume.

(a) Top

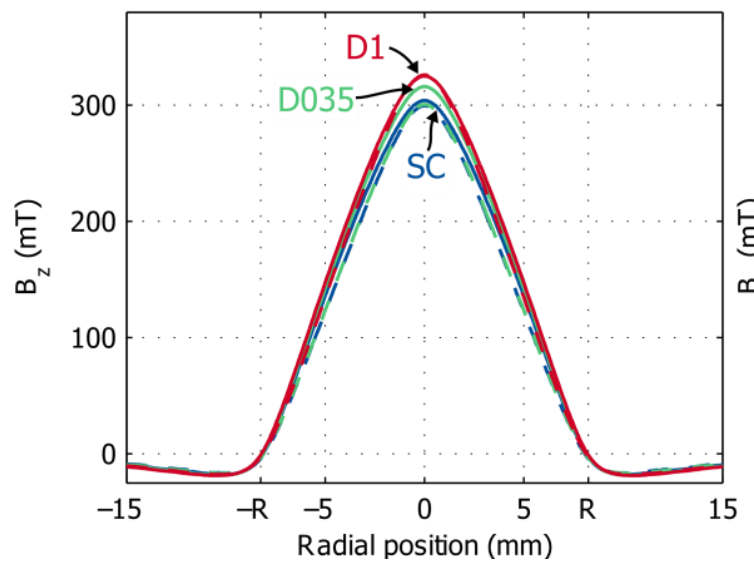

(b) Bottom

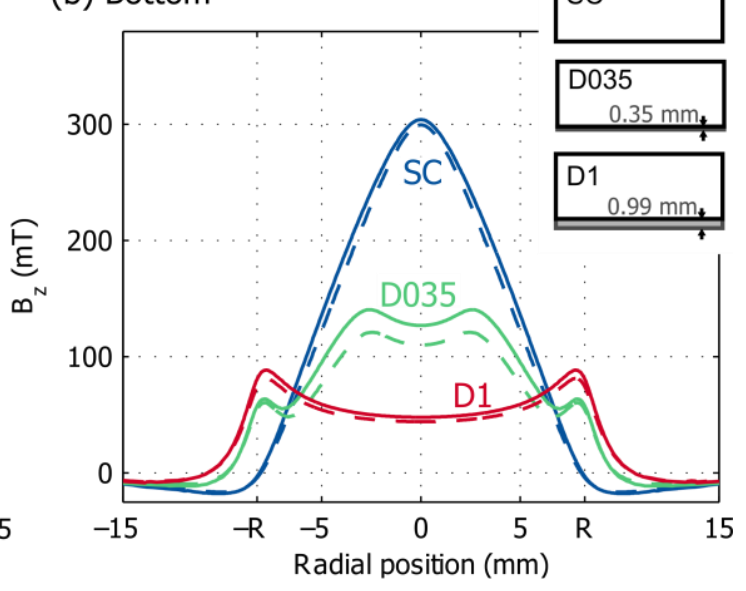

Figure 10 Comparison of modelling results of $B_{\mathrm{z}}$ above each surface of the SC/FM structure with a constant critical current density $J_{\mathrm{c} 2}$ (dashed line) and the original $J_{\mathrm{c}}(B)$ critical current density (continuous line). The SC, D035, and D1 configurations are shown in blue, green, and red, respectively.

It is of interest to investigate what happens when the magnetic field generated by the superconductor is much higher than the saturation magnetization of the ferromagnetic material, and to know if it is still useful to use iron in such high field environments. The model is therefore run with a higher (constant) critical current ten times larger than that used in the previous sections, i.e. $J_{c 2}^{\prime}=10 \times J_{c 2}=10.3 \times$ $10^{8} \mathrm{~A} / \mathrm{m}^{2}$. Note that the practical way of reaching such higher critical current density would be to simply decrease the operating temperature. Figure 11 shows the modelling results obtained at $J_{c}=J_{c 2}^{\prime}$ when the superconductor is combined with ferromagnetic discs of thicknesses $0.35 \mathrm{~mm}, 0.99 \mathrm{~mm}$, and $1.90 \mathrm{~mm}$ (the D035, D1, and D2 configurations, respectively); these are all made of Supra50 material (saturation magnetization $=1.4 \mathrm{~T}$ ). To achieve full penetration of the superconductor, a magnetizing induction of $15 \mathrm{~T}$ is applied.

As can be seen, the remanent magnetic flux density is approximately ten times higher, with maximum values on the order of 3 teslas. Nevertheless, the general behaviour is similar to the previously obtained results. The trapped field on the top face increases with increasing thickness of ferromagnet. The increase, however, is relatively less significant than found previously, e.g. for D2, the increase of $B_{\mathrm{Z}}^{\mathrm{c}}$ is $3.0 \%$, instead of $9.6 \%$ for the lower constant critical current density $J_{\mathrm{c} 2}$. On the bottom face, the distribution of $B_{\mathrm{z}}$ is still conical, but attenuated at its centre and widened out at the edges. The interesting feature of the results shown in figure 11 is that the previous conclusions are still valid. A thicker ferromagnet leads to a larger decrease on the bottom and a larger increase on the top. We also see that a higher flux needs a thicker ferromagnet of a given material to achieve an equivalent shielding effect. According to the rough estimation given by equation (3), the minimum thickness of ferromagnet above which it is no longer saturated $\left(d^{*}\right)$ is directly proportional to the superconductor critical current density $J_{\mathrm{c}}$. In the present case this would correspond to approximately ten times the 
value found for the initial $J_{\text {c }}$, i.e. $d^{*} \approx 10 \mathrm{~mm}$. In the practical thickness range investigated for figure $11(0.35$ to $2 \mathrm{~mm})$, the regime is such that the ferromagnet is driven to saturation, at least partially. This is evidenced from the results shown in figure 11(b) which show a gradual evolution of the flux profiles.

It is of interest to compare the contribution of the totally saturated ferromagnet to that of a permanent magnet with a uniform axial magnetization $M=M_{\text {sat }}=1.4 \mathrm{~T}$. On the symmetry axis, the contribution of the permanent magnet can be computed by the analytical formula $[54,61]$

$$
B_{\mathrm{z}}(z)=\frac{\mu_{0} M}{2}\left(\frac{z+L / 2}{\sqrt{(z+L / 2)^{2}+a^{2}}}-\frac{z-L / 2}{\sqrt{(z-L / 2)^{2}+a^{2}}}\right)
$$

where $a$ is the diameter of the sample, $L$ its height and $z$ the distance on the symmetry axis measured from the magnet centre. For the D2 configuration, the contribution of such a permanent magnet is found to be $59 \mathrm{mT}$ at the top Hall probe location. This increment is lower than the $86 \mathrm{mT}$ increase of $B_{\mathrm{z}}^{\mathrm{c}}$ observed in figure 11(a) for the same D2 configuration. Similar results are found for the two other configurations (D035 and D1) in figure 11. These results, although based on a simple analytical model, suggest that the field enhancement due to flux closure through the soft ferromagnet is not simply given by the addition of the superconductor field and the stray field of a permanent magnet which has the same size and magnetization saturation as the investigated ferromagnetic material.

The important conclusion to be drawn is that the ferromagnet still plays a role in increasing the field produced by the superconductor, even though the latter is twice the intrinsic saturation magnetization of the ferromagnetic material. Interestingly, the regime investigated is one for which magnetic flux density measured at some distance from the ferromagnet is extremely sensitive to the thickness of ferromagnet. Although this is out of the scope of the present study, this strong sensitivity to thickness could be usefully exploited to form the basis of a contactless thickness sensor of ferromagnetic materials.

(a) Top

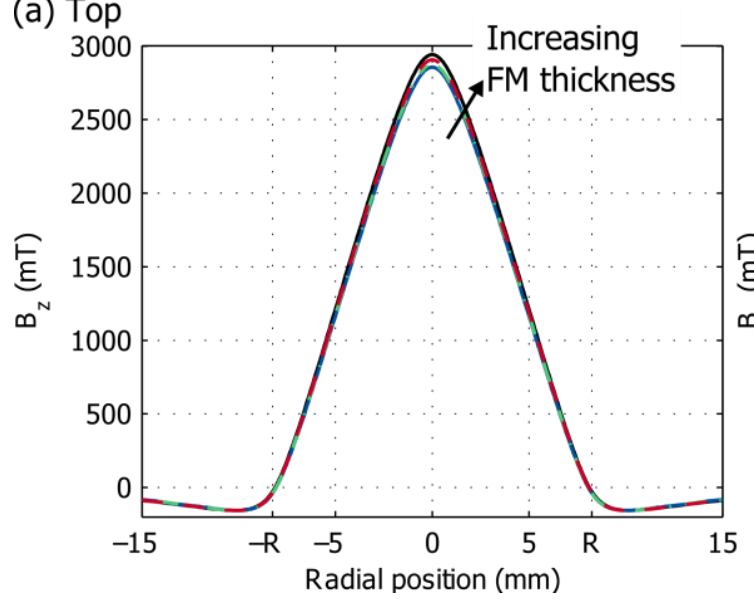

(b) Bottom

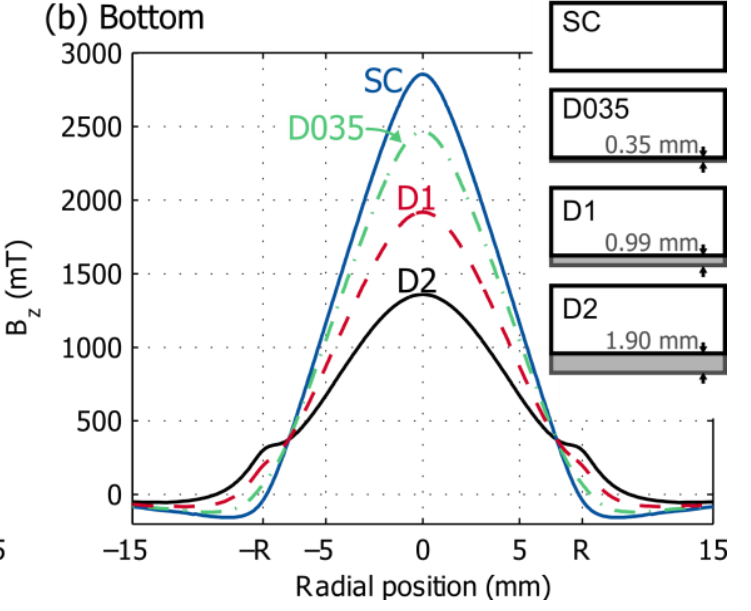

Figure 11 Modelling results of $B_{\mathrm{z}}$ above each surface of the SC/FM structure with a constant critical current density $J_{c 2}^{\prime}=10 \times J_{c 2}=10.3 \times 10^{8} \mathrm{~A} / \mathrm{m}^{2}$. The SC, D035, D1 and D2 configurations are shown in blue, green, red, and black, respectively. 


\section{Conclusion}

In this paper, the surface magnetic flux density above both faces of several superconductor/ferromagnet (SC/FM) hybrid structures has been measured. These results were then modelled using a 2D axisymmetric finite element method based on the $\boldsymbol{H}$-formulation. The models use the physical parameters of the SC and FM determined from independent characterization measurements carried out in a previous work. The modelling results are in excellent qualitative and quantitative agreement with the measurements, which validate the model for further investigations. The model is then used to study the magnetic flux distribution and predict the behaviour for other constitutive laws and geometries. We have investigated successively the influence of ferromagnet thickness and saturation magnetization, and the critical current density of the superconductor, including the influence of a field-dependent $J_{\mathrm{c}}(B)$. In all studied configurations, a ferromagnet section is attached to the bottom of a superconducting disc. At the bottom face, the ferromagnetic material acts as a magnetic shield, so that the flux density is reduced globally. Conversely, the flux density is enhanced on the top face (i.e. opposite to the ferromagnet) and the present investigations aimed at a better understanding of this phenomenon.

First, the results demonstrate that the thickness and saturation magnetization of the ferromagnetic material are important and play somewhat similar roles. The higher the saturation magnetization and/or the thicker the ferromagnetic material, the higher the trapped field on the top face and the larger the shielding effects at the bottom. A ferromagnet of given thickness below its full saturation will drive the majority of flux lines radially towards its sides, therefore shielding a large part of the magnetic induction $B_{z}$. If the trapped field is increased, the amount of diverted/shielded flux will not change after the full saturation of the ferromagnet. Therefore, the effect of the ferromagnet will become relatively less important as the generated flux increases.

Based on these considerations, the results show that the increase of magnetic flux vs. thickness of ferromagnetic disc exhibits a "kink" corresponding to full saturation of the ferromagnet. We have derived a simplified analytical model based on magnetic flux conservation to roughly estimate the most suitable ferromagnet thickness $d^{*}$, below which saturation occurs and above which weak thickness-dependence is observed. Remarkably, the rough estimation of $d^{*} \approx J_{\mathrm{c} 0} a^{2} / 6 M_{\text {sat }}$ agrees reasonably well with that obtained with accurate $2 \mathrm{D}$ finite element modelling of the full assembly. An increase of the ferromagnet thickness well beyond $d^{*}$ is of low interest. Therefore, this $d^{*}$ value can be used as a very useful "rule of thumb" to investigate the significance of the ferromagnet effects. Investigations for constant and field-dependent critical current densities lead to nearly similar results. This shows that the particular $J_{\mathrm{c}}(B)$ dependence is not a crucial parameter in investigating the behaviour of SC/FM structures. In addition, we show that the ferromagnet is also beneficial in improving the field gradient - and hence the magnetic levitation force - outside the superconductor.

The last and perhaps most relevant result of this study is that the flux density on the face opposite to the ferromagnet is shown to be enhanced by the ferromagnet, even though the generated field outside the assembly is much larger (twice in the present investigation) than the saturation magnetization of the ferromagnet. This was checked by running the models with a critical current density ten times higher, which is similar to lowering the temperature from $77 \mathrm{~K}$ to $20 \mathrm{~K}$ with the measured superconductor. Conversely, thin ferromagnetic discs can be driven to full saturation even though the outer magnetic field is much smaller (one fourth in the present investigation) than the saturation magnetization of the ferromagnet. These results aid significantly the understanding of the contribution of ferromagnetic sections to the trapped field produced by bulk high temperature superconductors, which is fundamental to realising practical applications of these technologically important materials. 


\section{Acknowledgments}

This work is part of an Action de Recherches Concertées grant from the Ministry of Higher Education through the Research Council of the University of Liege (ARC 11/16-03). Dr Mark Ainslie would like to acknowledge the support of a Royal Academy of Engineering Research Fellowship.

\section{References}

[1] Durrell J H, Dancer C E J, Dennis A, Shi Y, Xu Z, Campbell A M, Hari Babu N, Todd R I, Grovenor C R M and Cardwell D A 2012 Supercond. Sci. Technol. 25112002

[2] Yamamoto A, Ishihara A, Tomita M and Kishio K 2014 Appl. Phys. Lett. 105032601

[3] Yoshida T, Naito T and Fujishiro H 2015 IEEE Trans. Appl. Supercond. 256801204

[4] Fuchs G, Häßler W, Nenkov K, Scheiter J, Perner O, Handstein A, Kanai T, Schultz L and Holzapfel B 2013 Supercond. Sci. Technol. 26122002

[5] Tomita M and Murakami M 2003 Nature 421 517-20

[6] Durrell J H, Dennis A R, Jaroszynski J, Ainslie M D, Palmer K G B, Shi Y H, Campbell A M, Hull J, Strasik M, Hellstrom E E and Cardwell D A 2014 Supercond. Sci. Technol. 27082001

[7] Werfel F N, Floegel-Delor U, Rothfeld R, Riedel T, Goebel B, Wippich D and Schirrmeister P 2012 Supercond. Sci. Technol. 25014007

[8] Oka T, Fukazawa H, Fukui S, Ogawa J, Sato T, Ooizumi M, Tsujimura M and Yokoyama K 2014 Physica C 496 58-62

[9] Ainslie M D, Fujishiro H, Ujiie T, Zou J, Dennis A R, Shi Y H and Cardwell D A 2014 Supercond. Sci. Technol. 27065008

[10] Ainslie M D and Fujishiro H 2015 Supercond. Sci. Technol. 28053002

[11] Lousberg G P, Fagnard J F, Ausloos M, Vanderbemden P and Vanderheyden B 2010 IEEE Trans. Appl. Supercond. 20 33-41

[12] Lousberg G P, Fagnard J F, Chaud X, Ausloos M, Vanderbemden P and Vanderheyden B 2011 Supercond. Sci. Technol. 24035008

[13] Prat-Camps J, Sanchez A and Navau C 2013 Supercond. Sci. Technol. 26074001

[14] Granados X, Torner M, Puig T and Obradors X 2007 Applied Superconductivity, IEEE Transactions on 17 1629-32

[15] Hull J R and Strasik M 2010 Supercond. Sci. Technol. 23124005

[16] Takao T, Tanoue N, Kameyama S, Doi T and Kamijo H 2012 IEEE Trans. Appl. Supercond. 226187706

[17] Takao T, Horie T, Usami T, Takahashi M and Kamijo H 2014 IEEE Trans. Appl. Supercond. 243600504 
[18] Parks D, Weinstein R, Davey K, Sawh R P and Mayes B W 2009 IEEE Trans. Appl. Supercond. 19 2937-40

[19] Majoros M, Sumption M D and Collings E W 2009 IEEE Trans. Appl. Supercond. 19 3352-5

[20] Kovác P, Hušek I, Melišek T, Ahoranta M, Šouc J, Lehtonen J and Gömöry F 2003 Supercond. Sci. Technol. 16 1195-201

[21] Krüger P, Grilli F, Vojenciak M, Zermeño V M R, Demencik E and Farinon S 2013 Appl. Phys. Lett. 102202601

[22] Gömöry F, Vojenciak M, Pardo E, Solovyov M and Šouc J 2010 Supercond. Sci. Technol. 23 034012

[23] Vojenciak M, Šouc J and Gömöry F 2011 Supercond. Sci. Technol. 24075001

[24] Wen H, Bailey W, Goddard K, Al-Mosawi M, Beduz C and Yang Y 2009 IEEE Trans. Appl. Supercond. 19 1652-5

[25] Ainslie M D, Yuan W and Flack T J 2013 IEEE Trans. Appl. Supercond. 234700104

[26] Ainslie M D, Hu D, Zou J and Cardwell D A 2015 IEEE Trans. Appl. Supercond. 254602305

[27] Otabe E S, Nemoto S, Kiuchi M, Matsushita T, Hayashi T, Fujino K and Ni B 2010 J. Phys.: Conf. Ser. 234032046

[28] Tsuzuki K, Motohiro M, Felder B, Watasaki M, Kase S, Sato R and Izumi M 2013 IEEE Trans. Appl. Supercond. 236409414

[29] Philippe M P, Fagnard J F, Kirsch S, Xu Z, Dennis A R, Shi Y H, Cardwell D A, Vanderheyden B and Vanderbemden P 2014 Physica C 502 20-30

[30] Bean C P 1962 Phys. Rev. Lett. 8 250-3

[31] Bean C P 1964 Rev. Mod. Phys. 36 31-9

[32] Hari Babu N, Kambara M, Shi Y H, Cardwell D A, Tarrant C D and Schneider K R 2002 Supercond. Sci. Technol. 15 104-10

[33] Hari Babu N, lida K, Shi Y H and Cardwell D A 2006 Physica C 445-448 286-90

[34] Egan R, Philippe M, Wera L, Fagnard J F, Vanderheyden B, Dennis A, Shi Y, Cardwell D A and Vanderbemden P 2015 Rev. Sci. Instrum. 86025107

[35] Aperam Alloys Imphy, http://www.aperam.com/alloys-imphy/

[36] VACUUMSCHMELZE GmbH \& Co. KG, http://www.vacuumschmelze.com/

[37] Haindl S, Hengstberger F, Weber H W, Meslin S, Noudem J and Chaud X 2006 Supercond. Sci. Technol. 19108

[38] Cardwell D A, Murakami M, Zeisberger M, Gawalek W, Gonzalez-Arrabal R, Eisterer M, Weber H W, Fuchs G, Krabbes G, Leenders A, Freyhardt H C and Babu N H 2005 Supercond. Sci. Technol. 18 S173 
[39] Zou J, Ainslie M D, Hu D and Cardwell D A 2015 IEEE Trans. Appl. Supercond. 254900505

[40] Zhang M, Kvitkovic J, Pamidi S V and Coombs T A 2012 Supercond. Sci. Technol. 25 125020

[41] Patel A and Glowacki B A 2012 Supercond. Sci. Technol. 25125015

[42] Zou J, Ainslie M D, Fujishiro H, Bhagurkar A G, Naito T, Hari Babu N, Fagnard J F, Vanderbemden P and Yamamoto A 2015 Supercond. Sci. Technol. 28075009

[43] Ainslie M D, Rodriguez-Zermeno V M, Hong Z, Yuan W, Flack T J and Coombs T A 2011 Supercond. Sci. Technol. 24045005

[44] Ainslie M D, Flack T J and Campbell A M 2012 Physica C 472 50-6

[45] COMSOL Inc., http://www.comsol.com

[46] Kim Y B, Hempstead C F and Strnad A R 1962 Phys. Rev. Lett. 9 306-9

[47] Plummer C and Evetts J E 1987 IEEE Trans. Magn. 23 1179-82

[48] Rhyner J 1993 Physica C 212 292-300

[49] Xu Z 2012 Ph.D. thesis University of Cambridge

[50] Dewhurst C D, Lo W, Shi Y H and Cardwell D A 1998 Mater. Sci. Eng. B 53 169-73

[51] Lo W, Dewhurst C D, Cardwell D A, Vanderbemden P, Doyle R A and Astill D M 1996 Appl. Supercond. 4 507-17

[52] Eisterer M, Haindl S, Zehetmayer M, Gonzalez-Arrabal R, Weber H W, Litzkendorf D, Zeisberger M, Habisreuther T, Gawalek W, Shlyk L and Krabbes G 2006 Supercond. Sci. Technol. 19 S530

[53] Kii T, Kinjo R, Bakr M A, Choi Y W, Yoshida K, Ueda S, Takasaki M, Ishida K, Kimura N, Sonobe T, Masuda K and Ohgaki H 2011 Physica C 471 897-900

[54] Chen I G, Liu J, Weinstein R and Lau K 1992 J. Appl. Phys. 72 1013-20

[55] Lousberg G P, Fagnard J F, Haanappel E, Chaud X, Ausloos M, Vanderheyden B and Vanderbemden P 2009 Supercond. Sci. Technol. 22125026

[56] Hull J R 2000 Supercond. Sci. Technol. 13 R1

[57] Nishijima S, Eckroad S, Marian A, Choi K, Kim W S, Terai M, Deng Z, Zheng J, Wang J, Umemoto K, Du J, Febvre P, Keenan S, Mukhanov O, Cooley L D, Foley C P, Hassenzahl W V and Izumi M 2013 Supercond. Sci. Technol. 26113001

[58] Sotelo G G, De Oliveira R A H, Costa F S, Dias D H N, De Andrade R and Stephan R M 2015 IEEE Trans. Appl. Supercond. 253601005

[59] Ma K B, Postrekhin Y V and Chu W K 2003 Rev. Sci. Instrum. 74 4989-5017

[60] Brandt E H 1989 Science 243 349-55 
[61] Durand E 1968 Magnétostatique (Paris: Masson et Cie) 